\title{
INSTABILITY OF PERIODIC STATES FOR THE SIVASHINSKY EQUATION
}

\author{
BY \\ A. NOVICK-COHEN \\ Michigan State University, East Lansing, Michigan
}

\begin{abstract}
The Sivashinsky equation is an asymptotically derived model equation for evolution of the solid-liquid interface which occurs during directional solidification of dilute binary alloys. During the solidification process interfaces are known experimentally to yield planar, cellular, cusped, or dendritic structures. Cellular structures, interpreted here as periodic one dimensional nontrivial steady states, are shown in this paper to be unstable, if they exist, within the context of the Sivashinsky equation. Symmetric nontrivial steady states are likewise shown to be unstable.
\end{abstract}

1. Introduction. In this paper we discuss stability of periodic steady states for the one dimensional Sivashinsky equation

$$
\begin{aligned}
& u_{t}=\left(-u+\frac{1}{2} u^{2}-K u_{x x}\right)_{x x}-\alpha u \\
& u_{x}(0)=u_{x x x}(0)=0 \\
& u_{x}(L)=u_{x x x}(L)=0
\end{aligned}
$$

where $K$ and $\alpha$ are positive constants. Here $u(x, t)$ denotes the location of the solid-liquid interface relative to a planar interface which is moving at an externally imposed velocity $V$ which is the speed at which the sample is transported through a freezing temperature profile. Equation (1.1) was derived by looking at long wavelength instabilities in the limit in which the segregation coefficient is small [1]. (By examining alternative limits, it is possible to derive other equations, [9].)

For the purpose of applications it is important to be able to control the structure of the interface, since the shape of interface during freezing determines the microstructure of the resultant material. Experimentally one sees planar interfaces, cellular interfaces, cusped interfaces, cusped interfaces with droplet formation at the bottoms of the cusps, and dendritic interfaces. Since Eq. (1.1) gives the location of a single valued interface, droplet formation cannot be described within the context of this equation. Some results are known which are relevant to planar interfaces and cusped interfaces. In particular in [3] it was shown there that if the initial data is sufficiently small and if $\alpha$ is sufficiently large, or if $L$ is sufficiently small then solutions to (1.1) exist globally and decay exponentially toward the planar state. In

Received September 1, 1988.

This work was supported by the Air Force Office of Scientific Research, Grant \#87-0267.

(C) 1990 Brown University 
[2], it was shown that, for sufficiently large initial data, solutions grow unboundedly in $L_{2}$. For a different set of boundary data, the $L_{\infty}$ norm was seen to blow up in finite time for certain parameter values and sufficiently large initial data [2]. These two results appear to be related to the development of cusped interfaces of finite or infinite depth.

The question now remains as to whether there is any possibility of cellular interfaces within the framework of Eq. (1.1). We interpret cellular interfaces here as stable periodic steady states. The possibility of the existence of stable periodic structures is suggested by experiment and was thought to be indicated by the numerical calculations $[4,8]$. The following perturbation calculation also suggests that stable periodic nontrivial steady states might have been expected.

Consider a perturbation expansion in the neighborhood of $\alpha=\frac{1}{4} K^{-1}$. Set $\alpha=$ $\frac{1}{4} K^{-1}-\varepsilon$ and $u=\delta u^{0}(x)+\delta^{2} u^{1}(x)+\cdots$ where $\delta=\sqrt{|\varepsilon|}$. Substituting these assumptions into (1.1), setting $\tau=|\varepsilon| t$ and expanding eliminating resonant terms yields $u=\delta A \cos (x / \sqrt{2} K)-\frac{2}{9} \delta^{2} A^{2} \cos (x \sqrt{2} / K)$ where $A(\tau)$ satisfies

$$
A_{\tau}=\frac{\varepsilon}{|\varepsilon|} A+\frac{1}{18 K} A^{3} \text {. }
$$

Thus we might expect subcritical instabilities to stabilize into roll solutions if the subcritical branch bends back and stabilizes [8].

In the present paper we assume periodic nontrivial steady states to exist and prove that they are not stable. More precisely, we look at the associated eigenvalue problem and demonstrate the existence of an eigenfunction with positive (growing) eigenvalue. It is still possible that there are "long-lived" periodic transient states or nontrivial stable states which are not periodic. We do however demonstrate that there is no possibility of symmetric nontrivial stable steady states. The methods used here are taken from the calculus of variations and are reminiscent of those used by Chafee [5].

2. The eigenvalue problem. In [2] existence and uniqueness were discussed for solutions $u(x, t) \in C\left([0, T], H_{E}^{2}\right) \cap C\left((0, T), H_{E}^{4}\right)$ of the equations

$$
\begin{aligned}
& u_{t}=\left(-u+\frac{1}{2} u^{2}-K u_{x x}\right)_{x x}-\alpha u, \\
& u_{x}(0)=u_{x x x}(0)=0, \quad u_{x}(L)=u_{x x x}(L)=0
\end{aligned}
$$

where

$$
H_{E}^{2}=\left\{u(x, t) \in H^{2} \mid u_{x}(0)=u_{x}(L)=0\right\}
$$

and where

$$
H_{E}^{4}=\left\{u(x, t) \in H^{2} \mid u_{x x}(x, t) \in H_{E}^{2}\right\} .
$$

Suppose that $u_{0}(x)$ is some smooth $\left(H_{E}^{4}\right)$ periodic steady state of $(1.1)$. Then it must satisfy

$$
\begin{gathered}
\left(-u_{0}+\frac{1}{2} u_{0}^{2}-K u_{x x}\right)_{x x}-\left(\alpha u_{0}=0,\right. \\
u_{0 x}(0)=u_{0 x x x}(L)=0, \quad u_{0, x}(L)=u_{0 x x x}(L)=0 .
\end{gathered}
$$


Note further that upon integrating $(2.2)$ over $[0, L]$

$$
\bar{u}_{0}=\int_{0}^{L} u_{0}(x) d x=0 .
$$

Let us linearize about this steady state solution, $u=z+u_{0}$. Then

$$
\begin{aligned}
& z_{t}=\left(\left(-1+u_{0}\right) z-K z_{x x}\right)_{x x}-\alpha z, \\
& z_{x}(0)=z_{x x x}(0)=0, \quad z_{x}(L)=z_{x x x}(L)=0 .
\end{aligned}
$$

In this paper we prove that $u_{0}(x)$ is unstable in the following sense

THEOREM 2.1. If $u_{0}(x)$ is a smooth periodic nontrivial steady state solution then the associated eigenvalue problem

$$
\begin{gathered}
\lambda z=\left(\left(-1+u_{0}\right) z-K z_{x x}\right)_{x x}-\alpha z, \\
z_{x}(0)=z_{x x x}(0)=0, \quad z_{x}(L)=z_{x x x}(L)=0
\end{gathered}
$$

has at least one eigenfunction with a positive eigenvalue.

Our approach will be as follows. First, we consider a functional which serves as a Liapunov functional over a restricted class of initial data. Then, we note that all steady state solutions are critical points of this functional, i.e., the first variation vanishes. Next, we examine the second variation functional and isolate a perturbation $\eta_{0}$ in $H^{1}$ for which the second variation is negative at these periodic solutions. Afterwards, we return to consider the second variation functional and minimize over all possible perturbations subject to a restriction related to our isolated perturbation $\eta_{0}$. We then show that the minimizer to this second minimization problem turns out to be an eigenfunction of (2.5) which possesses a positive (growing) eigenvalue.

The proof of Theorem 2.1 is given in a series of lemmas. No explicit use will be made later of Lemma 2.2 which is included to motivate the importance of the functional $F(t)$.

LeMmA 2.2. If $\bar{u}(0)=(1 / L) \int_{0}^{L} u(x, 0) d x \geq 0$, then Eq. (1.1) possesses an associated Liapunov functional, $F(t)$.

Proof. Defining $w=u-\bar{u}$ where $\bar{u}(t)=(1 / L) \int_{0}^{L} u(x, t) d x$ Eq. (1.1) may be written as

$$
\begin{aligned}
& w_{t}=\left(-w+\frac{1}{2} w^{2}+w \bar{u}-K w_{x x}\right)_{x x}-\alpha w, \\
& w_{x}(0)=w_{x}(L)=0, \\
& w_{x x x}(0)=w_{x x x}(L)=0 .
\end{aligned}
$$

Define $v(x, t)$ to be the solution to $v_{x x}=w, v_{x}(0)=v_{x}(L)=0$, and $\int_{0}^{L} v(x) d x=$ 0 . Then multiplying both sides of $(2.6)$ by $\left(-w+\frac{1}{2} w^{2}+w \bar{u}-w_{x x}-\alpha v\right)$ and integrating by parts yields

$$
\frac{d}{d t} \int_{0}^{L}\left\{-\frac{1}{2} w^{2}+\frac{1}{6} u^{3}+\frac{1}{2} K w_{x}^{2}+\frac{1}{2} \alpha v_{x}^{2}\right\} d x+\int_{0}^{L} w w_{t} \bar{u} d x \leq 0 .
$$


Note from Eq. (2.1) that $\bar{u}(t)=\bar{u}(0) e^{-\alpha t}$. Thus integrating over $[0, T]$ and integrating by parts

$$
\begin{aligned}
\int_{0}^{L} & \left.\left\{-\frac{1}{2} w^{2}+\frac{1}{6} w^{3}+\frac{1}{2} K w_{x}^{2}+\frac{1}{2} \alpha v_{x}^{2}\right\} d x\right|_{0} ^{T}+\left.\bar{u} \int_{0}^{L} \frac{1}{2} w^{2} d x\right|_{0} ^{T} \\
& \leq-\int_{0}^{T} \int_{0}^{L} \frac{\alpha}{2} w^{2} \bar{u} d x d t .
\end{aligned}
$$

From (2.7) it follows that if $\bar{u}(0) \geq 0$ then

$$
F(t)=\int_{0}^{L}\left\{-\frac{1}{2} w^{2}+\frac{1}{6} w^{3}+\frac{1}{2} K w_{x}^{2}+\frac{1}{2} \alpha v_{x}^{2}+\frac{1}{2} \bar{u} w^{2}\right\} d x
$$

acts as a Liapunov functional for Eq. (2.1).

Of particular interest is the case $\bar{u}(0)=0$ which defines a positively invariant subspace $\bar{u}(t)=0$. All steady states belong to this subspace by virtue of (2.3) and our destabilizing perturbations will also belong to this same space. In short, it will be sufficient for instability to restrict ourselves to this subspace on which there is a Liapunov functional.

Lemma 2.3. The first variation (the Frechet derivative) of $F(t)$ vanishes when evaluated at steady states over perturbation in $H^{1}$ with zero mean.

Proof. If $\eta \in \bar{U}_{0}$, where $\bar{U}_{0}=\left\{\eta \in H^{1} \mid \int_{0}^{L} \eta(x) d x=0\right\}$ then

$$
\left(F\left(u_{0}+\delta \eta\right)-F\left(u_{0}\right)\right)=\delta \int_{0}^{L}\left\{-u_{0} \eta+\frac{1}{2} u_{0}^{2} \eta+K u_{0 x} \eta_{x}+\alpha v_{0 x} \tilde{\eta}_{x}\right\} d x+O\left(\delta^{2}\right),
$$

where $\tilde{\eta}$ is the solution to $\tilde{\eta}_{x x}=\eta, \tilde{\eta}_{x}(0)=\tilde{\eta}_{x}(L)=0$, and $\int_{0}^{L} \tilde{\eta} d x=0$. Thus integrating by parts

$$
F^{\prime}\left(u_{0}\right) \eta=\int_{0}^{L}\left\{-u_{0}-\frac{1}{2} u_{0}^{2}-K u_{0 x x}-\alpha v_{0}\right\} \eta d x
$$

where $v_{0}$ is the solution to $v_{0 x x}=u_{0}, v_{0 x}(0)=v_{0 x}(L)=0$, and $\int_{0}^{L} v_{0}(x) d x=0$. On the other hand, integrating (2.2) and using the boundary conditions and (2.3) yields

$$
-u_{0}+\frac{1}{2} u_{0}^{2}-K u_{0 x x}-\alpha v_{0}=k
$$

where $k$ is a constant. Thus $F^{\prime}\left(u_{0}\right) \eta=k \int_{0}^{L} \eta d x=0$ since $\eta$ has zero mean.

Since the first variation vanishes, the next natural question is to ascertain the behavior of the second variation.

Lemma 2.4. Suppose that $u_{0}(x)$ is a nontrivial steady state with period $L / N, N>$ 1 , then there exists a perturbation $\eta_{0} \in \bar{U}_{0}$ such that the second variation functional $\delta^{2} F\left(u_{0}, \eta_{0}\right)=\Lambda_{0}$ for some $\Lambda_{0}<0$.

REMARK. It follows that while periodic steady states are critical points of $F$, they are not even weak local minimizers. The proof of this lemma is similar to that of Theorem 8.2 in Carr, Gurtin, and Slemrod [6]. 
Proof. It is easy to calculate the second variation functional $V\left(u_{0}, \eta\right)$

$$
V\left(u_{0}, \eta\right)=\int_{0}^{L} \frac{1}{2}\left\{\left(-1+u_{0}\right) \eta^{2}+K \eta_{x}^{2}+\alpha \tilde{\eta}_{x}^{2}\right\} d x
$$

where $\tilde{\eta}$ satisfies $\tilde{\eta}_{x x}=\eta, \tilde{\eta}_{x}(0)=\tilde{\eta}_{x}(L)=0, \int_{0}^{L} \tilde{\eta} d x=0$. Since $u_{0}(x)$ is periodic with period $L / N, N>1$, it must repeat itself. Thus there exists an $L^{\prime}$ such that

$$
u\left(L^{\prime}\right)=u(0), \quad u_{0 x x}\left(L^{\prime}\right)=u_{0 x x}(0), \quad u_{0 x}\left(L^{\prime}\right)=u_{0 x x x}\left(L^{\prime}\right)=0
$$

and

$$
v_{0 x}\left(L^{\prime}\right)=\int_{0}^{L^{\prime}} u_{0}(x) d x=0
$$

Define

$$
\eta_{1}= \begin{cases}u_{0 x}(x), & 0 \leq x \leq L^{\prime} \\ 0, & L^{\prime} \leq x \leq L\end{cases}
$$

and define $\eta_{2}$ to be any function in $\bar{U}_{0}$ such that $\eta_{2}(0)=1$ and $\eta_{2}=0$ for $L^{\prime} \leq x \leq L$. Now let $\eta=\eta_{1}+\chi \eta_{2}$ where $\chi$ is a constant to be fixed. Noting that $\eta \in \bar{U}_{0}$, we substitute and calculate

$$
\begin{aligned}
V\left(u_{0}, \eta\right)= & \int_{0}^{L^{\prime}} \frac{1}{2}\left\{\left(-1+u_{0}\right) u_{0 x}^{2}+K u_{0 x x}^{2}+\alpha u_{0}^{2}\right\} d x \\
& +\chi \int_{0}^{L^{\prime}}\left\{\left(-1+u_{0}\right) u_{0 . x} \eta_{2}+K u_{0 x x} \eta_{2 x}+\alpha u_{0} \tilde{\eta}_{2 x}\right\} d x+O\left(\chi^{2}\right) .
\end{aligned}
$$

where $\tilde{\eta}_{2}$ satisfies $\tilde{\eta}_{2 x x}=\eta_{2}, \tilde{\eta}_{2 x}(0)=\tilde{\eta}_{2 x}(L)=0$, and $\int_{0}^{L} \tilde{\eta}_{2}(x) d x=0$. Since $u_{0}$ is a steady state, (2.9) holds. Differentiating (2.9) once,

$$
\left(-u_{0}+\frac{1}{2} u_{0}^{2}-K u_{0 x x}-\alpha v_{0}\right)_{x}=0
$$

multiplying by $u_{0 x}$ and integrating by parts yields that the first integral contribution must vanish. Integrating the remaining integral in (2.10) by parts and using (2.11) yields

$$
\begin{aligned}
V\left(U_{0}, \eta\right)= & \chi \int_{0}^{L^{\prime}}\left\{\left(-1+u_{0}\right) u_{0 x}-K u_{0 x x x}-\alpha v_{0 x}\right\} \eta_{2} d x \\
& +\left.\chi\left[K u_{0 x x} \eta_{2}+\alpha v_{0 x} \tilde{\eta}_{2 x}\right]\right|_{0} ^{L^{\prime}}=\chi\left[-K u_{0 x x}(0)\right] .
\end{aligned}
$$

Suppoe that $u_{0 x x}(0) \neq 0$, then we may pick $\chi=\chi_{0}$ so that if $\eta_{0}=\eta_{1}+\chi_{0} \eta_{2}$, then $V\left(u_{0}, \eta_{0}\right)=\Lambda_{0}<0$.

If $u_{0 x x}(0)=0$ then we must proceed to construct a new destabilizing perturbation as follows. Define

$$
\eta_{1}= \begin{cases}u_{0 . x x}(x), & 0 \leq x \leq L^{\prime} \\ 0, & L^{\prime} \leq x \leq L\end{cases}
$$


and define $\eta_{2}$ to be any function in $\bar{U}_{0}$ such that $\int_{0}^{L^{\prime}} u_{0 x}^{2} \eta_{2} d x \neq 0$ and such that $\eta_{2}=0$ for $x \in\left[L^{\prime}, L\right]$. Again we set $\eta_{0}=\eta_{1}+\chi \eta_{2}$, we note that $\eta_{0} \in \bar{U}_{0}$ and we evaluate

$$
\begin{aligned}
V\left(u_{0}, \eta\right)= & \int_{0}^{L^{\prime}} \frac{1}{2}\left\{\left(-1+u_{0}\right) u_{0 x x}^{2}+K u_{0 x x x}^{2}+\alpha u_{0 x}^{2}\right\} d x \\
& +\chi \int_{0}^{L^{\prime}}\left\{\left(-1+u_{0}\right) u_{0 x x} \eta_{2}+K u_{0 x x x} \eta_{2 x}+\alpha u_{0 x} \tilde{\eta}_{2 x}\right\} d x+O\left(\chi^{2}\right) .
\end{aligned}
$$

This time we multiply (2.2) by $u_{0 x x}$ and integrate by parts to find that the first integral vanishes. Integrating the second integral by parts,

$$
\begin{aligned}
V\left(u_{0}, \eta\right) & =\chi \int_{0}^{L^{\prime}}\left\{\left(-1+u_{0}\right) u_{0 x x}-K u_{0 x x x x}-\alpha u_{0}\right\} \eta_{2} d x+O\left(\chi^{2}\right) \\
& =-\chi \int_{0}^{L^{\prime}} u_{0 x}^{2} \eta_{2} d x+O\left(\chi^{2}\right) .
\end{aligned}
$$

Since by assumption $\int_{0}^{L^{\prime}} u_{0 x}^{2} \eta_{2} d x \neq 0, \chi=\chi_{0}$ can be chosen so that if $\eta_{0}=$ $\eta_{1}+\chi_{0} \eta_{2}$, then $V\left(u_{0}, \eta_{0}\right)=\Lambda_{0}<0$.

RemarK. Note that if we work on the interval $[-L, L]$ then solutions which start symmetric must stay symmetric, i.e., $u(-x, 0)=u(x, 0) \Rightarrow u(-x, t)=u(x, t)$ for $t>0$. In particular, if a steady state is approached as $t \rightarrow \infty$, then $u_{0}(-L)=u_{0}(L)$, $u_{0 x}(-L)=u_{0 x}(L)=0$ and $v_{0 x}(L)=\int_{0}^{L} u_{0}(x) d x=0$. Reviewing the necessary steps in the previous lemma, this together with Lemmas 2.6 and 2.7 which follow, yield

Lemma 2.5. If the initial data is symmetric then there are no nontrivial stable steady states.

Remark. Note that $\eta_{0}$ cannot be expected to satisfy the boundary conditions of (2.2). In order to find the appropriate eigenfunction we resort to variational techniques. We define problem $\mathrm{P}\left(u_{0}, \eta_{0}\right)$.

$\mathrm{P}\left(u_{0}, \eta_{0}\right):$ Minimize the functional

$$
A(\eta)=2 V\left(u_{0}, \eta\right)=\int_{0}^{L}\left\{\left(-1+u_{0}\right) \eta^{2}+K \eta_{x}^{2}+\alpha \tilde{\eta}_{x}^{2}\right\} d x
$$

over the class $\eta \in \bar{U}_{0}$, subject to the constraint $B(\eta)=\int_{0}^{L} \tilde{\eta}_{x}^{2} d x=\int_{0}^{L} \tilde{\eta}_{0 x}^{2} d x$.

Lemma 2.6. Problem $\mathrm{P}\left(u_{0}, \eta_{0}\right)$ has a solution and the solution satisfies a twice integrated version of the associated eigenvalue problem (2.5) in a weak sense. Lemma 2.6 is proved below for the sake of completeness.

Proof. The proof of Lemma 2.5 consists in demonstrating that the conditions of Theorem 6.3.2 (Berger [7]) hold. For convenience, we quote Theorem 6.3.2:

Theorem. Let $A$ and $B$ be $C^{1}$ functionals on $X$ where $X$ is a reflexive Banach space and suppose

(i) $A$ is w.l.s.c. and coercive on $X \cap\{B(\eta) \leq$ constant $\}$

(ii) $B$ is weakly continuous

(iii) $B(0)=0, B^{\prime}(\eta)=0$ only at $\eta=0$. 
Then the equation $A^{\prime}(\eta)=\lambda B^{\prime}(\eta)$ has a one parameter family of nontrivial solutions $\left(\eta_{R}, \lambda_{R}\right)$ for all $R \neq 0$ in the range of $B(\eta)$ and $B(\eta)=R$. Moreover $\eta_{R}$ can be characterized as the function which minimizes $A(\eta)$ over the set $B(\eta)=R$.

We take as our space $X$ the space $\bar{U}_{0}$. Since $\bar{U}_{0}$ is a closed subset of $H^{1}$ which is itself a reflexive Banach space, it follows that $\bar{U}_{0}$ is reflexive. The norm on $\bar{U}_{0}$ will be again the $H^{1}$ norm. Furthermore, it is easy to check that $A$ and $B$ are $C^{1}$ functionals. In particular

$$
\begin{aligned}
& \frac{1}{2} A^{\prime}(\eta)=\left(-1+u_{0}\right) \eta-\eta_{x x}-\alpha \eta^{2} \\
& \frac{1}{2} B^{\prime}(\eta)=\tilde{\eta} .
\end{aligned}
$$

To check that $A$ is w.l.s.c. we write $A=A_{1}+A_{2}$ with

$$
A_{1}(\eta)=\int_{0}^{L}\left\{\left(-1+u_{0}\right) \eta^{2}+\alpha \tilde{\eta}_{x}^{2}\right\} d x \quad \text { and } \quad A_{2}(\eta)=\int_{0}^{L} K \eta_{x}^{2} d x
$$

Suppose $\eta_{i}-\bar{\eta}$ in $H^{1}$, then since $L_{2}$ is compactly imbedded in $H^{1}, \eta_{i} \rightarrow \bar{\eta}$ in $L_{2}$. Using Poincaré's inequality for functions with zero mean we see that $A_{1}(\eta)$ is continuous in $L_{2}$ hence w.l.s.c. Again using Poincaré's inequality for functions with zero mean we have that $A_{2}(\eta)$ is equivalent to the $H^{1}$ norm. Since the $H^{1}$ norm is w.l.s.c. it follows that $A_{2}$ is also.

To check the coercivity of $A(\eta)$ we see by Lion's Lemma [7] that

$$
\int_{0}^{L}-\left(1-u_{0}\right) \eta^{2} d x \leq \sup \left|-1+u_{0}\right| \int_{0}^{L} \eta^{2} d x \leq \frac{1}{2} K \int_{0}^{L} \eta_{x}^{2} d x+C(K) \int_{0}^{L} \tilde{\eta}_{x}^{2} d x
$$

hence

$$
A(\eta) \geq \frac{1}{2} K \int_{0}^{L} \eta_{x}^{2} d x+(\alpha-C(K)) \int_{0}^{L} \tilde{\eta}_{x}^{2} d x .
$$

Since $\int_{0}^{L} \tilde{\eta}_{x}^{2} d x$ remains bounded if $B(\eta) \leq$ constant, clearly $A(\eta) \rightarrow \infty$ as $\|\eta\|_{H^{1}} \rightarrow$ $\infty$ so $A(\eta)$ is indeed coercive.

To check that $B(\eta)$ is weakly continuous we again use the argument that if $\eta_{i} \rightarrow \eta_{0}$ in $H^{1}$ then from the compact imbedding of $H^{1}$ in $L_{2}$ it follows that $\eta_{i} \rightarrow \eta_{0}$ in $L_{2}$. Moreover using Poincaré's inequality it is easy to check that $B(\eta)$ is continuous in $L_{2}$ and weak continuity is obtained.

Lastly, clearly if $\eta=0$ then $\tilde{\eta}_{x}=0$ hence $B(0)=0$. And if $B^{\prime}(\eta)=\tilde{\eta}=0$, then from the definition $\tilde{\eta}_{x x}=\eta$ it follows that $\eta=0$. Thus conditions of the theorem are satisfied and we obtain that for any $R=B(\eta)$

$$
\left(-1+u_{0}\right) \eta_{R}-K \eta_{R x x}-\alpha \tilde{\eta}_{R}=\lambda_{R} \tilde{\eta}_{R}
$$

where $\eta \in H^{1}$. In particular there is such a solution with $\bar{R}=\int_{0}^{L} \tilde{\eta}_{0 x}^{2} d x$.

$$
\left(-1+u_{0}\right) \eta-K \eta_{x x}-\alpha \tilde{\eta}=\lambda \tilde{\eta} .
$$

Lemma 2.7. The solution $\eta$ from the previous lemma is actually a classical solution of (2.13) and solves the associated eigenvalue problem (2.5). Its eigenvalue is positive.

Proof. The regularity of $\eta$ follows from the regularity of $u_{0}(x)$ together with classical bootstrapping arguments. Thus it is possible to differentiate (2.13) twice 
and it is seen to be a solution of the associated eigenvalue problem (2.5) if we can show that the boundary conditions are satisfied. It suffices to prove that $\eta_{x}(0)=$ $\eta_{x}(L)=0$, since by differentiating $(2.10)$ once the boundary conditions at $\eta_{x x x}(0)$ and $\eta_{x x x}(L)$ can then be seen to be automatically satisfied. In order to prove that the boundary conditions are satisfied we choose a function $\bar{\eta} \in \bar{U}_{0}$, such that $\bar{R}=$ $B(\bar{\eta})=\int_{0}^{L} \bar{\eta}_{0 x}^{2} d x$, which vanishes at $x=L$ but not at $x=0$. Then since $\eta$ minimizes $A(\eta)$ over the set $B(\eta)=\bar{R}$, it must also minimize $J(\eta)=A(\eta)+\lambda B(\eta)$ over the set $B(\eta)=\bar{R}$, hence

$J(\eta+\delta \bar{\eta})-J(\eta)=2 \delta \int_{0}^{L}\left\{\left(-1+u_{0}\right) \eta \bar{\eta}+K \eta_{x} \bar{\eta}_{x}+\alpha \tilde{\eta}_{x} \tilde{\bar{\eta}}_{x}+\lambda \tilde{\eta}_{x} \tilde{\bar{\eta}}_{x}\right\} d x+O\left(\delta^{2}\right) \geq 0$.

Integrating by parts and using (2.13) it follows that

$$
J(\eta+\delta \bar{\eta})-J(\eta)=2 \delta \eta_{x}(0) \bar{\eta}(0)+O\left(\delta^{2}\right) \geq 0 .
$$

Since (2.14) must hold for both $\delta$ positive and $\delta$ negative, it follows that $\eta_{x}(0)=0$. A parallel argument gives that $\eta_{x}(L)=0$.

Lastly, to complete the proof of Theorem 2.1, multiply (2.13) by $\eta$ and integrate by parts,

$$
\int_{0}^{L}\left\{\left(-1+u_{0}\right) \eta^{2}+K \eta_{x}^{2}+\alpha \tilde{\eta}_{x}^{2}\right\} d x=-\lambda \int \tilde{\eta}_{x}^{2} d x=-\lambda \bar{R}^{2}
$$

Since $\eta$ minimizes $A(\eta)$ relative to $B(\eta)=\bar{R}$, it follows that $A(\eta) \leq A\left(\eta_{0}\right)$ or equivalently that $-\lambda \bar{R} \leq \Lambda_{0}<0$. Therefore $\lambda$ must be positive.

Conclusion. The possibility of stable periodic steady states for the Sivashinsky equation is examined. It is demonstrated that if nontrivial periodic steady states do exist then they are not stable. It is also shown that nontrivial symmetric steady states are not stable.

\section{REFERENCES}

[1] G. I. Sivashinsky, On cellular instability in the solidification of a dilute binary alloy; Physica 8D. 243-248 (1983)

[2] A. Novick-Cohen, Blow up and growth in the directional solidification of dilute binary allol's. preprint

[3] C. Elliott and S. Zheng. On the C'ahn-Hilliard equation. Arch. Rational Mech. Anal. 96. 339-357 (1986)

[4] G. W. Young and S. H. Davis, Systems with small segregation coefficient, Phys. Rev. B 34. 33883396 (1986)

[5] N. Chafee, Asymptotic behavior for solutions of a one-dimensional parabolic equation with homogeneous Neumann boundary conditions. J. Differential Equations 18, 111-134 (1975)

[6] J. Carr, M. E. Gurtin, and M. Slemrod, Structural phase transitions on a finite interval. Arch. Rational Mech. Anal. 86, 317-351 (1984)

[7] M. S. Berger, Nonlinearity and Functional Analysis, Academic Press. New York. 1977

[8] G. W. Young. S. H. Davis, and K. E. Brattkus, Anisotropic interface kinetics and tilted cells in unidirectional solidification. J. Crystal Growth 83, 560-571 (1988)

[9] K. Brattkus and S. H. Davis, Cellular growth near absolute stability, Phys. Rev. B38, 11.452-11.460 (1988) 